\title{
Methods and tools for high and system level synthesis
}

\author{
Prihozhy A. \\ State University of Informatics and Radioelectronics, Computer \\ Systems Department \\ 6 P.Brovki Str., Minsk 220027, Belarus, \\ Phone: 0172649486 - Fax: 0172310914 \\ e-mail: csd@cc.bsuir.minsk.by
}

\begin{abstract}
This paper presents new methods for high- and system-level synthesis based on transformation of a behavioral description to a special behavioral model, development of new and modification of existing synthesis techniques for this model, development of net-based synthesis model and techniques extending target architectures. VHDL-based high-level synthesis tools running on an IBM PC platform are described.
\end{abstract}

\section{Keywords}

High-level synthesis, system-level synthesis, net-based synthesis, transformat-ions, scheduling, allocation, binding, VHDL

\section{INTRODUCTION}

Effective high-level synthesis systems include ALERT, AMICAL, CATHEDRAL, CMUDA, DAA, ELLA, FACET, HAL, HIS, MAHA, MIMOLA, PSAL2, Yorktown Silicon Compiler, and others (Camposano,1989, Courtois,1994, Gajski,1992, 1994, Goossens,1989, Jerraya,1993, Mcfarland,1990, Mermet,1993, Vercest, 1990). They develop synthesis methodology consisting of the following tasks:

- compiling a behavioral description presented in a hardware description language to an intermediate format

- generating the control (CFG) and data (DFG) flow graphs

- scheduling the description operators and statements

- allocation of the functional, storage, and interconnection units 
- binding the behavior constructions to the units

- generating the data path (DP) and finite state machine (FSM).

These systems usually accept the behavioral description as it was defined by a designer, therefore the description expresses rather a designer's point of view than target architecture requirements. The synthesis tasks to be solved are very complex combinatorial problems in this case, and it is very difficult or impossible to find the optimal design. Natural extensions of high-level synthesis are system level synthesis and low power asynchronous circuit synthesis (Asynchronous,1994, Mermet,1997).

This paper presents new methods and tools for high- and system-level synthesis that explore behavioral description transformation, synthesis techniques based on orthogonality analysis, and net-based synthesis techniques. Section 2 introduces the concepts underlying the new synthesis methodology. Section 3 describes behavioral description transformation rules that explore a special behavioral model. Scheduling methods are presented in section 4 . Section 5 presents allocation and binding methods for the model. Section 6 describes the key concepts of net-based synthesis methodology. The results for the AHILES high-level synthesis system appear in section 7 .

\section{SYNTHESIS METHODOLOGY}

The methodology is based on the following three main principles:

- transforming the source behavioral model to a special model, allowing efficient synthesis of high quality RTL-structures

- development and use of new analyzing, scheduling, allocation, binding, data path generation, and finite state machine generation techniques that explore the special model advantages

- extending traditional high-level synthesis methodology in order to automatically design and optimize asynchronous circuits and systems.

Behavioral description transformations have been locally used in several highlevel synthesis systems (Camposano,1989, Gajski,1992,1994, Jerraya,1993, Mcfarland,1990, Mermet,1993). This methodology employs transformations to obtain the preliminary defined special behavioral model that speeds up the design process and allows generating faster and cheaper designs for the same constraints on design parameters. Various HDLs constructions introduced to represent a behavior have been investigated to find a representation that increases design space exploration freedom and allows development of efficient scheduling, allocation, and binding techniques. It has been found out that the main restrictions on the order of computations are implied by CFG. The main idea of the special model is to replace the order implied by CFG with a weaker order implied by DFG. This can be achieved by splitting control structures into many separate parts connected 
through data dependencies. The developed transformation rules increase freedom for reordering statements and extend the design space.

Synthesis from the special behavioral model. The special behavioral model decreases the influence of control structures on the order of calculations. The order is defined through using orthogonal relations introduced for Boolean and bit signals and variables, if-then statements, and operators. The orthogonal statements can share the same functional unit and execute in one FSM state concurrently. Due to the orthogonality relations, the number of statements executed in one HLFSM state increases and the number of states decreases. Techniques which perform variable lifetime analysis and calculate the statements precedence and compatibility relations are modified to account for the special model and orthogonality relations. Due to introducing the probability for a variable to take value true (1), the overall execution time under constraints on resources is estimated and minimized. Scheduling, allocation, binding, and DP and FSM generation methods are modified and extended in order to

- explore orthogonality between statements

- minimize the execution time mathematical expectation.

Net-based high-and system-level synthesis. Net-based synthesis methodology extends the set of target architectures. It constitutes a theoretical and practical basis for design of asynchronous circuits and systems. The key concept of this methodology is a net schedule which concurrency level, execution time, and cost are defined through the set of concurrent statement pairs. Net-based scheduling, allocation, and binding methods and techniques explore the concurrency space and generate net schedules for given constraints on design parameters. The net schedule can be used for synthesis of circuits and systems directly or can be a source for generating sequential schedules.

\section{BEHAVIORAL MODEL TRANSFORMATION}

Source behavioral model. The behavioral specification is described in VHDL (IEEE,1988). Modelling the behavior is in many aspects the same as in the traditional high-level synthesis systems (Mermet,1993). The behavioral description is presented by object declarations, process, signal and variable assignment, if, case, loop, exit, next, and wait statements. The design specification may be composed of several VHDL units and libraries.

Special behavioral model. The special behavioral model is described through using a subset of VHDL statements. The wait, signal assignment, variable assignment, loop, exit, and next statements may execute unconditionally and conditionally. The loop statement has no the iteration scheme. The behavioral description CFG constructed of these statements has only one segmented path. Each segment may be processed separately. This allows the development of efficient lifetime analysis, 
scheduling, allocation, and binding techniques. The behavioral model for GCD (Mermet,1993) is shown in Figure 1.

Transition probabilities. Probability $p(v)$ is introduced for each conditional variable or signal $v$. This is the probability of event for object $v$ to take value true (1). The probability of event for object $v$ to take value false(0) is equal to $1-p(v)$.

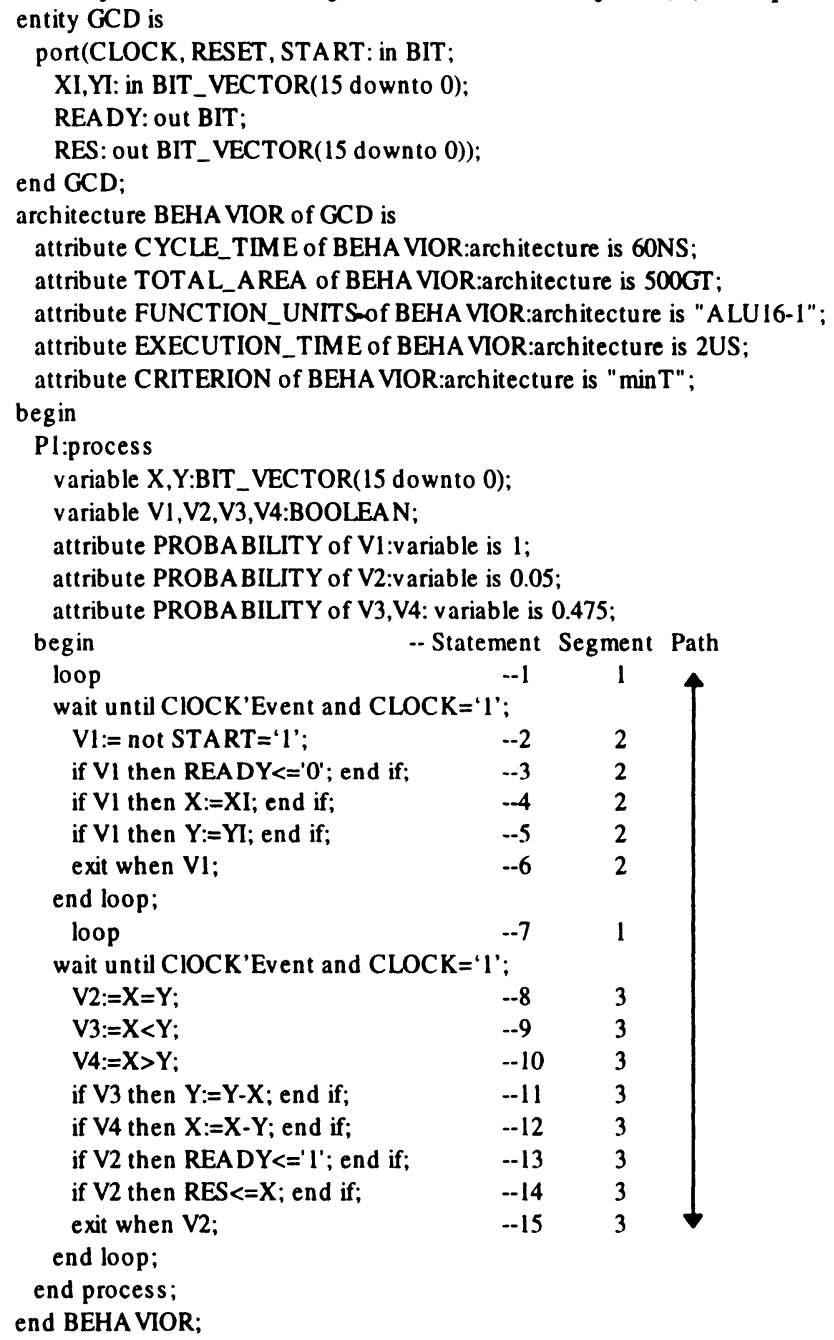

Figure 1 GCD special behavioral model

If condition $v 1$ or...or $v n=1$ is true for objects $v 1, \ldots, v n$ then $p(v 1)+\ldots+p(v n)=1$. The transition probabilities are defined by attributes as it is shown in Figure 1.

Optimization problem. Two formulations of the optimization problem can be specified. One formulation minimizes the design execution time mathematical expectation with constraints on the design cost. Another formulation minimizes the 
design cost with a constraint on the execution time. Additional constraints are the bounding clock cycle time, number of functional units, and others. The optimization problem is described in VHDL by attribute declarations and specifications (Figure 1).

Functional unit description. The set of VHDL operators is partitioned into subsets of compatible operators or operators that may be introduced into FSM. Including an operator into a subset depends on the operands width. For each operator and functional unit the delay, area, and number of pipeline stages are defined. All the values are represented by formulas.

Transformation rules. A source VHDL behavioral description is equivalently transformed to the special model by applying transformation rules. These modify the behavior CDFG to speed up the design process and to improve the design parameters. The rules transform a loop statement with the iteration scheme to a loop without the scheme, reorder independent and dependent statements, insert a statement into if- and loop-statements, extract computations from if-statement, split if-statement into separate parts, transform if-statement to a logical expression and variable assignment statement, merge exit-statements, unroll loop-statement without the iteration scheme.

\section{SCHEDULING FOR THE SPECIAL BEHAVIORAL MODEL}

Background. Efficient scheduling techniques include as soon as possible (ASAP), as late as possible (ALAP), list scheduling, freedom-based scheduling, forcedirected scheduling, integer linear programming formulation (ILPF), dynamic loop scheduling, path-based scheduling, scheduling for pipelined architectures, and others (Camposano,1989, Gajski,1992,1994, Goossens,1989, Hwang,1991, Jerraya,1993, Mcfarland,1990, Mermet,1993). They accept the behavioral description in a general form.

Single-path-based scheduling. The novel scheduling method performs equiva-lent transformation of the source behavioral description. The transformation results in the special behavioral model which CFG has only one segmented path. The extended scheduling techniques use relations constructed on the sets of signals, variables, operators, and statements.

Segment tree. The segment tree is a hierarchical structure of the special behavioral model CFG. The tree root is the process statement. The other non-terminal nodes are loop statements. The terminal nodes are sequential statements of the model. The loop or process body statements constitute a segment. The FSM states are introduced during top down traversal of the segment tree.

Orthogonality and implication analysis for signals and variables. Orthogonal-ity, implication, and independence matrix $\boldsymbol{R}$ 's rows and columns correspond to Boolean and bit signals and variables. Matrix elements $r_{i j}$ belong to set $\{\perp, \rightarrow, \leftarrow$, $\leftrightarrow,-\}$ where $\perp$ defines objects $i$ and $j$ to be orthogonal, $\rightarrow$ defines object $i$ to imply 
object $j, \leftarrow$ defines object $j$ to imply object $i, \leftrightarrow$ defines objects $i$ and $j$ to be equivalent, '-' defines objects $i$ and $j$ to be independent. The matrix is generated during analysis of relational operators and inferring new relations when rules apply to operators and, or, xor, nand, nor, and not.

Orthogonality of statements. Two conditional statements if $c 1$ then Pl; end if; and if $c 2$ then P2; end if; are defined to be orthogonal iff $c 1$ and $c 2$ are orthogonal. The orthogonal statement bodies are mutually exclusive and may execute on the same functional unit.

Operators compatibility and proximity. There are two cases for operators to be compatible within one high-level finite state machine (HLFSM) state:

- the operators belong to orthogonal statements

- the operators are relational and have the same inputs.

The proximity accounts for the statement common inputs and outputs and is used to select compatible operators to be merged. Maximizing the operators proximity implies minimizing the number of interconnection units.

Statements precedence relation. Statements precedence relation $\boldsymbol{P R E}$ is union VALUUSE $\cup W A T$ of three subrelations. Relation VAL defines statement $i$ to precede statement $j$ if $i$ and jare not orthogonal and $i$ has an output value which is an input for $j$. Relation $U S E$ accounts for a variable may not be assigned a new value while the old value is still used. Relation WAT defines all the non-orthogonal statements located before a wait statement to precede the wait, and the wait statement to precede all the nonorthogonal statements located after it.

Scheduling for the special behavioral model. Scheduling techniques ASAP, ALAP, list scheduling, and others are extended for single-path-based scheduling. Given a special behavioral model, transition probabilities, and optimization task, the goal is to introduce states, distribute the statements onto the states, and gene-rate an appropriate schedule. To minimize the total execution time is to minimize the number of the HLFSM states and execution probability for each state.

HLFSM state execution probability. The state can include several if-then statements. If conditional objects $c 0, \ldots, c n$ are used in the state then execution probability $P($ state $)$ is estimated involving probabilities $p(c 0), \ldots, p(c n)$ and relations between the objects. For the orthogonality relation $P($ state $)=p(c 0)+\ldots+p(c n)$. For the independence relation $P($ state $)=1-(1-p(c 0)) * \ldots *(1-p(c n))$. For implication relation $c 0 \rightarrow, \ldots, \rightarrow c n$ the probability is $P($ state $)=p(c n)$. In general, the state probability is defined by a composition of these formulas.

Estimating the execution time. The overall execution time mathematical expectation is $T=C y c l e \_T i m e^{*} M_{1}$ where $M_{1}$ is the total number of states needed to execute the model. The number of states needed to execute segment $i$ is $M_{i}=M_{i}^{\text {body }}$ * $\left(1-P_{i}^{\text {exit }}\right) / P_{i}^{\text {exit }}+M_{i}^{\text {exit }}$ where $M_{i}^{\text {body }}$ and $M_{i}^{\text {exit }}$ are the number of states in the segment and before exit, and $P_{i}^{\text {exit }}$ is the probability to exit from the segment. 


\section{ALLOCATION AND BINDING FOR THE SPECIAL BEHAVIORAL MODEL}

Background. Effective allocation techniques include rule-based expert systems, greedy iterating, branch and bound, clique-partitioning, linear-programming, simulated-annealing-based, path-based, force-directed, graph coloring, and interactive allocation algorithms (Camposano,1989, Gajski,1992,1994, Hwang, 1991, Jerraya,1993, Mcfarland,1990, Mermet,1993).

Single-path-based allocation and binding. Allocation and binding methods developed for the special behavioral model use the model advantages. The singlepath-based allocation and binding flow is as follows. First, the special behavioral model DFG is generated. Using the CFG single path, the variable lifetimes are computed and the variables compatibility is determined. The compatibility analysis is performed accounting for the orthogonality analysis and scheduling results. Using the variables, operators, and statements compatibility, the functional, storage, and interconnection units are allocated. Each variable, operator, and statement is binded to a unit in such a way as to minimize the DP and FSM cost.

$D F G$ weighed with relations. The special behavioral model DFG is defined as $D F G=(N, A)$ where $N=V \cup P t \cup L t \cup R$ is the set of nodes, $A=N x(V \cup P t \cup R)$ is the set of edges, $V$ is the set of variables, $P t$ is the set of ports, $L t$ is the set of literals, and $R$ is the set of statements. Variables compatibility relation $C v$ and statements compatibiity relation $\mathrm{Cr}$ are appended to DFG and used to fold DP.

Reenumeration of the HLFSM states. The HLFSM states are introduced and enumerated during the top down traversal of the segment tree and scheduling the segments. To define the variable lifetime by a state interval, the states must be reenumerated in the order for which the tree leaves are looked through from the left to the right. The new enumeration corresponds to the top down traversal of the states, but not the segments.

Lifetime analysis. Function $\operatorname{Inc}(v, s)$ defines the mode of use of variable $v$ in state $s$; Inc: $V \times S \rightarrow\{\varnothing,\{$ in $\},\{$ out $\},\{$ in,out $\}\}$ where $V$ is the set of variables, $S$ is the set of states, and in and out are the modes of using a variable. The variable $v$ lifetime is defined by interval $\left[s_{v}{ }^{\text {min }}, s_{v}{ }^{\text {max }}\right]$ where the bounding states are determined through using the segment tree structure and the orthogonality relations.

Compatibility of variables. Variables used within one state constitute set $V^{v}$ and are implemented as a wire. Variables used in several states constitute set $V^{m}$ and are allocated on a latch, register, RAM, and ROM. Variables of set $V^{m}$ may be merged if they are compatible. Variables $v 1$ and $v 2$ are compatible if their lifetime intervals are not crossed or each statement that contains $v l$ is orthogonal to each statement that contains $v 2$. The variables compatibility is described by matrix $C_{v^{\circ}}$. Compatibility of statements. Compatibility of statements implies compatibility of operators that is derived from the operators orthogonality, their execution in the same or different states, and the operation's ability to share resources. The operators compatibility relation is $C_{R}=(\operatorname{Shan}-(S t a \mid O r t))$ Sin where Sha is the 
relation built of the pairs of operators which allow sharing resources, Sta is the relation built of the pairs of operators executed in the same state, Ort is the operators orthogonality relation, and Sin is the relation built of the pairs of relational operators that execute in the same state and have the same input values.

Reordering operator inputs. The goal is to decrease the design cost by increasing the number of data dependences that can be allocated on the same interconnection unit.

The data path optimization problem is formulated as

$$
\min _{d p \in D P} \alpha_{f}{ }^{*} c_{d p}^{f u}+\alpha_{s}{ }^{*} c_{d p}^{s t}+\alpha_{c}{ }^{*} c_{d p}^{i c}
$$

where DP is the set of feasible data paths; $C_{d p}{ }^{f u}, C_{d p}{ }^{s t}$, and $C_{d p}{ }^{i c}$ are the functional, storage, and interconnection unit costs; $\alpha_{\rho}, \alpha_{s}$, and $\alpha_{c}$ are factors.

Folding techniques. Depending on the design space exploration approach, the DP optimization techniques are partitioned into global and local (Mcfarland, 1990). Both of them can fold the DP graph by merging variables, operators, and data dependences. The global techniques minimize the target function by searching for variables and operators to be merged, and by reordering operation inputs. The local techniques search for pairs of variables and operators to be preferably merged step by step.

\section{NET-BASED SYNTHESIS}

Background. The Petri net is a concurrency model widely used for representing asynchronous behavior of processes (Petri,1962).

Principles. There are two main problems in synthesis of circuits and systems composed of variable execution time components, how to

- perform the scheduling, allocation, and binding tasks to optimize the design

- build the components, synthesize the control, and construct a system.

Both the problems can be solved within high-level synthesis net-based methodology (Prihozhy,1996).

Net schedule. The key concept of net-based synthesis is a net schedule that describes mixed sequential/concurrent execution of the statements. The noncyclic net schedule is directed graph $G_{H}=(N, H)$ where $N=\{1, \ldots, n\}$ is a set of statement numbers and $H$ is a statements direct precedence relation. The relation defines direct predecessors of each statement that executes when execution of all the predecessors is complete. Direct data dependences between the statements constitute the precedence relation defining the maximum concurrency net schedule. Two statements are sequential if a path of graph $G_{H}$ between the statements exists, otherwise the statements are concurrent. Statements are mutually exclusive if they are orthogonal or sequential. Mutually exclusive statements can never execute simultaneously. If mutually exclusive statements may share the same resources they are compatible. Matrix $\boldsymbol{Q}$ describes the data dependences 
between the statements which element $q_{i j}$ equals $l$ if $j$ uses a value delivered by $i$, and equals 0 otherwise. Matrix $W$ 's element $w_{i j}$ equals 0 if statements $i$ and $j$ may not execute on the same functional unit, equals $l$ if the statements may share resources, and equals 2 if the statements are orthogonal. Zero elements of matrix $\boldsymbol{Q}$ define maximum set $D_{M}$ of the concurrent statement pairs. Set $D=D_{M}$ defines the net schedule of maximum concurrency. The schedule execution time is defined by the cliques of graph $G_{D}=(N,-D)$ and the schedule cost is defined by the cliques of graph $G_{D}=(N, D)$.

Net scheduling and allocation. For any subset $D$ of set $D_{M}$ we search for a net schedule of less concurrency. Two optimization tasks are possible in order to

- minimize the net schedule execution time for constraint $S_{o}$ on the cost

- minimize the net schedule cost for constraint $T_{o}$ on the execution time.

One method solves the first task consecutively adding pairs to set $D$. Another method solves the second task consecutively removing pairs from set $D$ starting with set $D_{M}$. To select a pair to be added or removed, clique sets for graphs $\mathrm{G}_{D}$ and $\mathrm{G}_{D}$ are analyzed. The pairs that decrease the execution time and dose not increase the cost are the most preferable. When a pair is added to or removed from set $D$ the clique sets are recalculated. Adding pairs to $D$ is complete if the extended set implies cost $S$ greater than bounding cost $S_{o}$. Moving pairs from set $D$ is complete if the reduced set implies time $T$ greater than bounding time $T_{\sigma}$. We can use net scheduling for Petri nets as well.

Existence problem. The problem is formulated as to find out whether any net 


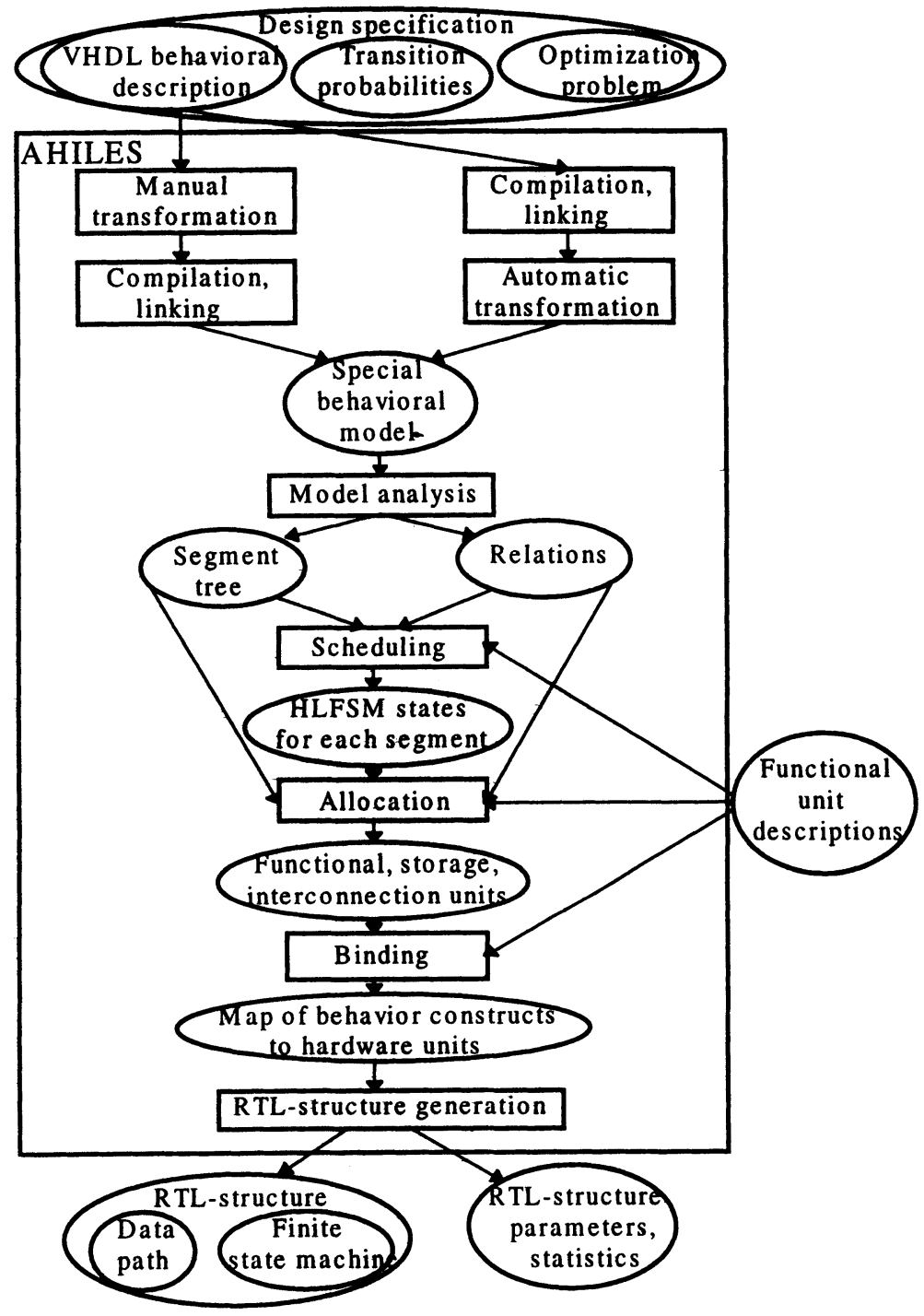

Figure 2 AHILES design flow

schedule is possible for given set D or not. In (Prihozhy,1996) the problem is reduced to solving a combined logical equation. The method proposed to solve the equation labels a graph in such a way as to avoid conflicts.

Net-based scheduling. The net schedule can be a source for synthesizing sequential schedules. An ordinary sequential schedule is generated by ASAP and ALAP techniques if the statement execution time equals the clock cycle time. A sequential schedule with chaining is generated by the list scheduling and other techniques for constraints on the cost or on the number of functional units. A sequential schedule with multicycling is generated if the functional unit execution time is 
greater than the clock cycle time. If functional units are functionally pipelined, statements have to be splitted into parts, one for each stage of the pipeline.

Asynchronous circuit and system synthesis. Synthesis of asynchronous circuits and systems is based on net scheduling algorithms, net allocation algorithms for functional, storage, and interconnection units, net binding algorithms, methods of constructing asynchronous circuit and system components, methods of synthesis of asynchronous circuits and systems composed of these components.

\section{RESULTS}

The described models, methods, techniques, and algorithms are realized within the AHILES high-level synthesis system (Figure 2) (Prihozhy,1996). Results generated for five benchmarks (Courtois,1994,Mermet,1993) are presented in Tables 1 and 2. All the RTL-structures are synthesized on a PC 486/50. Table 1 presents CPU time for synthesis steps. The VHDL compiler throughput is 100 to 280 lines per second. The overall synthesis time is 5 to $14 \mathrm{sec}$. Generated RTLstructure parameters appear in Table 2. The internal form size is 1.4 times greater than the VHDL-text size for behavioral descriptions and 0.86 times less for structural descriptions. AHILES introduced few FSM states for all the designs. This is due to the preliminary transformation of the behavioral descriptions, special behavioral model, and novel scheduling, allocation, and binding techniques. The average execution time of generated net schedules is $18 \%$ less than the execution time of optimal sequential schedules.

Table 1 Synthesis time (sec), PC 486/50

\begin{tabular}{lccccc}
\hline \multicolumn{1}{c}{$\begin{array}{c}\text { Synthesis } \\
\text { steps }\end{array}$} & Bubble & Gcd & Gcdf & Kalman & Pid \\
\cline { 2 - 6 } & 0.71 & 0.49 & 0.77 & 1.48 & 1.27 \\
\hline Compilation & 044 & 0.44 & 0.50 & 0.49 & 0.44 \\
Linking & 0.66 & 0.49 & 0,55 & 0.77 & 0.72 \\
Diagnostics & 0.87 & 0.66 & 0,66 & 4.57 & 1.09 \\
Analyzing & 0.55 & 0.44 & 0.44 & 0.77 & 0.61 \\
Scheduling & 0.88 & 0.55 & 0.55 & 1.37 & 1.04 \\
Allocation and binding & 1.10 & 1.05 & 0.99 & 1.65 & 1.38 \\
Data path generation & 0.60 & 0.55 & 0.49 & 0.83 & 0.66 \\
FSM generation & 0.60 & & & &
\end{tabular}

Table 2 Design parameters

\begin{tabular}{lccccc}
\hline \multirow{1}{*}{ Parameter } & \multicolumn{5}{c}{ Benchmarks } \\
\cline { 2 - 6 } & Bubble & Gcd & Gcdf & Kalman & Pid \\
\hline Behavior VHDL text (lines) & 119 & 50 & 60 & 220 & 180 \\
Behavior VHDL text (bytes) & 3009 & 2089 & 2844 & 7966 & 9978 \\
Behavior internal form (bytes) & 10148 & 7160 & 7512 & 19478 & 13680 \\
Statements & 79 & 19 & 29 & 176 & 122
\end{tabular}




\begin{tabular}{lccccc} 
Objects & 46 & 15 & 19 & 122 & 75 \\
CFG and DFG (bytes) & 5171 & 1409 & 1987 & 12340 & 8152 \\
FSM states & 20 & 2 & 5 & 16 & 23 \\
FSM transitions & 31 & 4 & 9 & 29 & 33 \\
ALUs & 0 & 1 & 1 & 1 & 1 \\
Functional units width (bits) & 0 & 16 & 32 & 17 & 32 \\
Registers & 7 & 2 & 2 & 18 & 13 \\
Register width (bits) & 104 & 32 & 64 & 138 & 389 \\
RAMs & 1 & 0 & 0 & 3 & 0 \\
ROMs & 0 & 0 & 0 & 3 & 1 \\
Collectors & 0 & 0 & 0 & 5 & 9 \\
Multiplexers & 4 & 4 & 4 & 14 & 8 \\
Multiplexer width (bits) & 68 & 64 & 128 & 155 & 227 \\
Multiplexer inputs & 13 & 8 & 8 & 36 & 33 \\
DP internal form (bytes) & 6177 & 3031 & 3170 & 17075 & 12425 \\
FSM internal form (bytes) & 4522 & 1132 & 1532 & 9927 & 8197 \\
Structure internal form (bytes) & 10699 & 4163 & 4702 & 27002 & 20622 \\
Structure VHDL text (lines) & 416 & 164 & 184 & 1000 & 724 \\
Structure VHDL text (bytes) & 12383 & 4647 & 5241 & 31550 & 22938 \\
\hline
\end{tabular}

\section{ACKNOWLEDGMENTS}

The author gratefully acknowledge Luc Claesen, Bernard Courtois, Jean Mermet, Yervant Zorian, and other colleagues for collaboration.

\section{REFERENCES}

Asynchronous Circuits and Systems (1994) IEEE Design \& Test of Computers, 12, Summer.

Camposano, R. and Rosenstiel, W. (1989) Synthesizing Circuits from Behavioral Descriptions. IEEE Transactions, CAD-8, 171-80.

Courtois, B. (1994) CAD and Testing of ICs and Systems: Where are We Going? Journal of Microelectronic Systems Integration, 2, 139-201.

Gajski, D., Dutt, N.D., Wu, A., Lin, S. (1992) High-Level Synthesis: Introduction to Chip and System Design. Kluwer Academic Publishers, Norwell, Mass.

Gajski, D. and Ramachandran, L. (1994) Introduction to High-Level Synthesis. IEEE Design \& Test of Computers, 12, Winter, 44-54.

Goossens, G., Vandewalle, J., De Man, H. (1989) Loop Optimization in Register Transfer Scheduling for DSP-Systems, in Proc. 26th Design Automation Conference, 826-31. 
Hwang, T.H., Lee, J.H., Hsu, Y.C. (1991) A Formal Approach to the Scheduling Problem in High-Level Synthesis. IEEE Transactions, CAD-10, 464-75.

IEEE Standard VHDL Language Reference Manual (1988) IEEE Std. 1076-1987, IEEE, New York.

Jerraya, A.A., Park, I., O'Brien, K. (1993) Amical: An Interactive High-Level Synthesis Environment, in Proc. EDAC'93, IEEE Computer Society Press, Los Alamitos, Calif.

Mcfarland, M.C., Parker, A.C., Camposano, R. (1990) The High-Level Synthesis of Digital Systems. Proc.IEEE, 78, 301-36.

Mermet, J. ed. (1993) Fundamentals and Standards in Hardware Description Languages. Kluwer Academic Publishers, the Netherlands.

Mermet, J. and Nebel, W. ed. (1997) Low Power Design in Deep Submicron Electronics. Kluwer Academic Publishers.

Petri, C. (1962) Fundamentals of a Theory of Asynchronous Information Flow, in Proc. Congress, Amsterdam, 386-90.

Prihozhy, A. (1996) Net Scheduling in High-Level Synthesis. IEEE Design \& Test of Computers, 13, Spring, 26-35.

Prihozhy, A. (1996) Use of VHDL-Based Design Methodology and AHILES System for Education in Belarus, in Proc. Europ. Workshop on Microelectr. Education (ed. G. Kamarinos, N. Guillemot., and B. Courtois), World Scientific, Singapore, 217-20.

Verkest, D., Claesen, L., De Man, H. (1990) Correctness Proof of Parameterized Hardware Modules in the Cathedral-II Synthesis Environment, in Proc. European Design Automation Conference, Glasgow.

\section{BIOGRAPHY}

Anatoly Prihozhy is associate professor in the Computer Systems Department at the State University of Informatics and Radioelectronics of Belarus. Previously he worked as a principal researcher at the National Academy of Sciences. His interests include VHDL, logic-, high-, and system-level synthesis, design methodology, and tools. Prihozhy received a DiplIng in electrical engineering form the State Polytechnic and a Ph.D in computer sciences from the Academy of Sciences. He is a member of the IEEE Computer Society and an active member of the New York Academy of Sciences. Prihozhy heads the VHDL Users Group in Belarus. 